\title{
INFLUENCIA DE LA GRANULOMETRIA DE LA MUESTRA EN LA DISCRIMINACIÓN DE ESPECIES DE Salix POR INFRARROJO CERCANO
}

\section{INFLUENCE OF SAMPLE GRANULOMETRY ON DISCRIMINATION OF Salix SPECIES BY NEAR INFRARED}

\author{
Silvana Nisgoski ${ }^{1}$, Mayara Elita Carneiro ${ }^{2}$, Graciela Inés Bolzon de Muñiz ${ }^{1, \wedge}$
}

\begin{abstract}
RESUMEN
En Brasil, las especies cultivadas del género Salix son de gran importancia en la artesanía, con propiedades diferentes en relación a la maleabilidad y resistencia. Este trabajo tuvo como objetivo aplicar la espectroscopía de infrarrojo cercano (FT-NIR) para la discriminación de cuatro especies de Salix, comparando muestras de materia maciza y partículas. Las muestras de Salix viminalis, Salix x rubens, Salix purpurea y Salix sp., fueron seleccionadas en siete sitios en la región del Valle del Río Canoas, Estado de Santa Catarina (Brasil). Los espectros de absorción se obtuvieron en FT-NIR espectrofotómetro (Bruker Tensor 37). La forma y la granulometría de las muestras de Salix spp. tienen influencia en la discriminación de las especies por FT-NIR. La técnica tuvo mayor capacidad de distinción para la granulometría de menor tamaño.
\end{abstract}

Palabras clave: Espectroscopía de infrarrojo cercano, granulometría de la muestra, mimbre.

\begin{abstract}
In Brazil, the cultivated species of genus Salix have great importance in crafts, with different properties in relation to malleability and resistance. This paper aimed to apply near infrared spectroscopy (FT-NIR) for discrimination of four Salix species, comparing solid sample and particulate material. Samples from Salix viminalis, Salix x rubens, Salix purpurea and Salix sp. were collected in seven sites in the region of Rio Canoas Valley, Santa Catarina State, Brazil. It was obtained absorbance spectra in FT-NIR (Bruker Tensor 37). The shape and particle size of Salix spp. influenced the discrimination of species by near infrared. The technique had a more efficient distinction for smaller particle size samples.
\end{abstract}

Keywords: Near infrared spectroscopy, particle size sample, willow.

\footnotetext{
Dra. Eng. Florestal. Laboratório de Anatomia e Qualidade da Madeira, Departamento de Engenharia e Tecnologia Florestal, Universidade Federal do Paraná.

Curitiba,PR, Brasil. silvana.ufpr@gmail.com; graciela.ufpr@gmail.com

${ }^{2}$ Dra. Eng. Industrial Madeireira. Laboratório de Anatomia e Qualidade da Madeira, Departamento de Engenharia e Tecnologia Florestal,

Universidade Federal do Paraná. Curitiba, PR, Brasil.

^Corresponding author : gbmunize@ufpr.br

Received: 15.08.2013 Accepted: 13.05. 2014
} 


\section{INTRODUCCIÓN}

El género Salix, pertenece a la familia Salicaceae, y es importante desde el punto de vista económico ya que a partir de él se obtiene el ácido acetil salicílico, base de diversos analgésicos (Souza y Lorenzi 2005). Además, se utiliza como alimento para fauna silvestre, paisajismo, fijación de carbono, producción de fibras vegetales para uso en bioenergía (Zasada et al. 2002, Urso y Paiero 2008) y también para recuperación de suelos contaminados (Vervaeke et al. 2003, Punshon et al. 2003), entre otros.

En Brasil, las especies cultivadas, conocidas como "vime" (mimbre), son muy utilizadas en artesanía, siendo la región de la Sierra Catarinense, en el sur del país, la mayor productora de ramas, con cosecha anual de aproximadamente 16 mil toneladas, con un procesamiento local de sólo 1500 toneladas. El resto de la materia prima se comercializa en otros municipios y Estados, que representan aproximadamente 6 millones de reales brasileños por cosecha (cerca de 16 millones de dólares americanos).

La evaluación realizada por los extensionistas de la Empresa de Pesquisa Agropecuaria e Extensión Rural de Santa Catarina (EPAGRI 2006) han concluido que cuatro especies tienen potencial para uso en muebles y artesanías, que son: Salix purpurea con baja productividad, baja ramificación, color rojizo con interesante efecto estético y un excelente material para la artesanía; Salix x rubens, especie vigorosa y tolerante a las plagas y enfermedades, pero con flexibilidad limitada, que es de difícil trabajabilidad según los artesanos; Salix viminalis con menor ramificación y uniformidad en la longitud de las ramas, tarda más en producir ramas y las plantas son menos vigorosas y más susceptibles a las plagas; Salix sp., variedad poco estudiada, conocida popularmente como goma de mimbre, descripción realizada por Nascimento (2009) y Vargas et al. (2012).

Nascimento (2009), que estudió los aspectos de producción para artesanías en mimbre, sugiere que se debe considerar en primer lugar el tipo de producto que se pretende realizar, es decir, la respuesta que cada material tiene al esfuerzo propuesto. La opinión de los artesanos, por orden de preferencia, es: Salix sp. es la especie que mejor responde a los esfuerzos de flexión y torsión, sin embargo, sus ramas son cortas, muy ramificadas y no tienen un buen rendimiento en los cultivos experimentales. En el caso de Salix purpurea, el material es más homogéneo, las ramas resisten mejor a la torsión y flexión, con pequeño índice de estallido, además de presentar buenos resultados en cultivos experimentales, con ramas largas, poca ramificación y un adecuado factor de forma. Salix viminalis en comparación con las otras especies, tiene muchas ramas agrietadas longitudinalmente, sin llegar a romper, comprometiendo así la calidad de la pieza final. La especie que fue mal evaluada por los artesanos fue Salix x rubens, porque el material se agrieta en sentido longitudinal a las fibras, también es más duro y difícil de trabajar.

Vargas et al. (2012) evaluando las propiedades físicas y mecánicas de las cuatro especies concluyeron que todas pueden ser usadas para artesanía, entretanto, Salix viminalis y Salix purpurea son mas difíciles de trabajar.

Los aspectos externos de las especies son muy similares, por lo tanto es importante una correcta identificación para aplicaciones de mayor calidad, rendimiento y menor costo. La espectroscopía de infrarrojo cercano puede ser una herramienta rápida y de bajo costo, ya que es un análisis no destructivo aplicados en las industrias, laboratorios y en las líneas de producción, para la caracterización de propiedades morfológicas, químicas, físicas y mecánicas de los materiales lignocelulósicos (Schimleck 2008, Schimleck et al. 2009, Tsuchikawa y Schwanninger 2013).

Los estudios existentes de espectroscopía de infrarrojo están relacionados con la composición química del material, siendo conocida también la influencia de la preparación y el tamaño de partícula (Aucott et al. 1988, Pasikatan et al. 2001, Otsuka 2004, Blanco y Peguero 2008), dimensiones y dirección del corte anatómico en muestras de madera maciza (Faix y Böttcher 1992, Jones et al. 2006, Poke y Raymond 2006). 
Existen algunos estudios sobre la discriminación de diferentes tipos de madera, muestras del barreno de Pressler, virutas o en polvo (Adedipe et al. 2008, Russ et. al 2009, Sandak et al. 2011, Casale et al. 2010, Pastore et al. 2011, Braga et al. 2011), pero los estudios del efecto del tamaño de partícula de la muestra en la distinción de las especies son escasos. Las diferencias en las mediciones en el infrarrojo entre madera maciza y las partículas pueden ser el resultado de interacción entre la luz y el tipo de muestra, resultando en informaciones adicionales, y que puede sufrir influencia de la estructura superficial del lugar de la lectura, lo que contribuye a la aparición de ruidos en el espectro (Hein et al. 2010).

Por lo tanto este estudio tuvo por objetivo evaluar la espectroscopía de infrarrojo cercano para la discriminación de cuatro especies de Salix, comparando muestras maciza y aserrín.

\section{MATERIALES Y MÉTODOS}

Fueron utilizadas las especies Salix viminalis L. (35 muestras), Salix x rubens Schrank (25 muestras), Salix purpurea Linné (34 muestras) y Salix sp. (23 muestras) colectadas de acuerdo con la disponibilidad, en siete sitios en la región del valle del Río Canoas, Estado de Santa Catarina (Tabla 1), totalizando 117 muestras. De cada sitio fueron extraídas, aleatoriamente, 5 ramas, sin ramificaciones laterales, de 5 diferentes plantas, con longitudes y diámetros variados, todos con un año de edad.

Tabla 1. Características de la región de colecta de cuatro especies de Salix.

\begin{tabular}{lcccc}
\hline \multicolumn{1}{c}{ Municipio } & Sitio & Latitud Sur & Longitud Oeste & Altitud (m) \\
\hline Bocaina do Sul & 1 & $27^{\circ} 44^{\prime} 40^{\prime \prime}$ & $49^{\circ} 56^{\prime} 40^{\prime \prime}$ & 860 \\
Río Rufino & 2 & $27^{\circ} 51^{\prime} 39^{\prime \prime}$ & $49^{\circ} 46^{\prime} 44^{\prime \prime}$ & 860 \\
Lages & 3 & $27^{\circ} 48^{\prime} 57^{\prime \prime}$ & $50^{\circ} 19^{\prime} 33^{\prime \prime}$ & 916 \\
Lages & 4 & $27^{\circ} 48^{\prime} 57^{\prime \prime}$ & $50^{\circ} 19^{\prime} 33^{\prime \prime}$ & 916 \\
Río Rufino & 5 & $27^{\circ} 51^{\prime} 39^{\prime \prime}$ & $49^{\circ} 46^{\prime} 44^{\prime \prime}$ & 860 \\
Río Rufino & 6 & $27^{\circ} 51^{\prime} 39^{\prime \prime}$ & $49^{\circ} 46^{\prime} 44^{\prime \prime}$ & 860 \\
Urubici & 7 & $28^{\circ} 00^{\prime} 54^{\prime \prime}$ & $49^{\circ} 35^{\prime} 31^{\prime \prime}$ & 915 \\
\hline
\end{tabular}

Los espectros de absorbancia fueron obtenidos directamente de las ramas, totalizando 234 repeticiones, siendo efectuadas dos lecturas por muestra, una en cada superficie transversal del material, siendo utilizado el promedio de los análisis. Posteriormente, el material fue molido en un molino de cuchillas tipo Willey y tamizado. La terminología polvo fino se refiere a las partículas que quedaron retenidas en el tamiz de 60 mesh, y polvo grueso en el de 40 mesh. Fueron obtenidos 10 espectros por muestra, por especie y por sitio, en un total de 280 lecturas, siendo utilizado el promedio de los análisis.

El equipamiento utilizado fue un espectrofotómetro FTIR Tensor 37, marca Bruker, operando en el rango del infrarrojo cercando entre 4000 y $10000 \mathrm{~cm}^{-1}$. El Análisis de Componentes Principales (PCA) fue efectuado con el auxilio del software Unscrambler ${ }^{\circledR}$, versión 10 (CAMO Software). La obtención de los espectros y análisis fue de acuerdo a la norma ASTM E1655-05 (ASTM 2012) (Standard Practices for Infrared Multivariate Quantitative Analysis). Las muestras fueron secas al aire e permanecieron en cámara climatizada con temperatura de $20 \pm 3^{\circ} \mathrm{C}$ y humidad relativa de $65 \pm 1 \%$. 


\section{RESULTADOS Y DISCUSIÓN}

En la Figura 1 están representados los espectros NIR promedios para las cuatro especies de Salix estudiadas, con diferentes tipos de muestreo, los cuales tienen la misma tendencia y con perfiles de bandas características de materiales lignocelulósicos (Schimleck et al. 2009, Tsuchikawa y Schwanninger 2013).

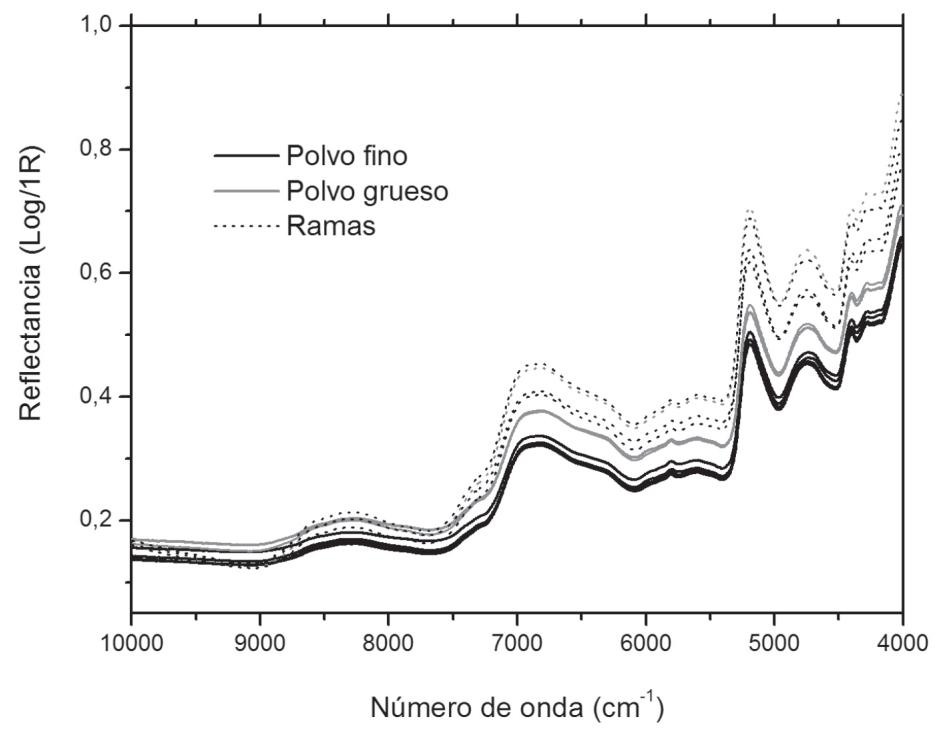

Figura 1. Espectros promedios FT-NIR para las cuatro especies de Salix en diferentes granulometrías.

Los picos observados entre 7000 y $5200 \mathrm{~cm}^{-1}$, son causados por las vibraciones de estiramiento de los enlaces $-\mathrm{OH}$ y $-\mathrm{CH}$, presentes en la celulosa y lignina. El pico en $4700 \mathrm{~cm}^{-1}$ corresponde al estiramiento -OH y a la deformación-CH atribuidas a las moléculas de agua (Tsuchikawa y Siesler 2003, Siesler et al. 2002).

Para una mejor interpretación, los espectros NIR fueron asociados a la técnica multivariada de análisis por componentes principales (PCA). Inicialmente fue realizado un PCA, de análisis cualitativo, para cada conjunto de muestreo (polvo fino, polvo grueso y ramas) para verificar la posibilidad de discriminación de las especies. Los espectros fueron tratados con la $2^{\text {a }}$ derivada (Savitzky y Golay 1964), para mejorar la calidad de la señal. La Figura 2, muestra los modelos para las diferentes granulometrías. 

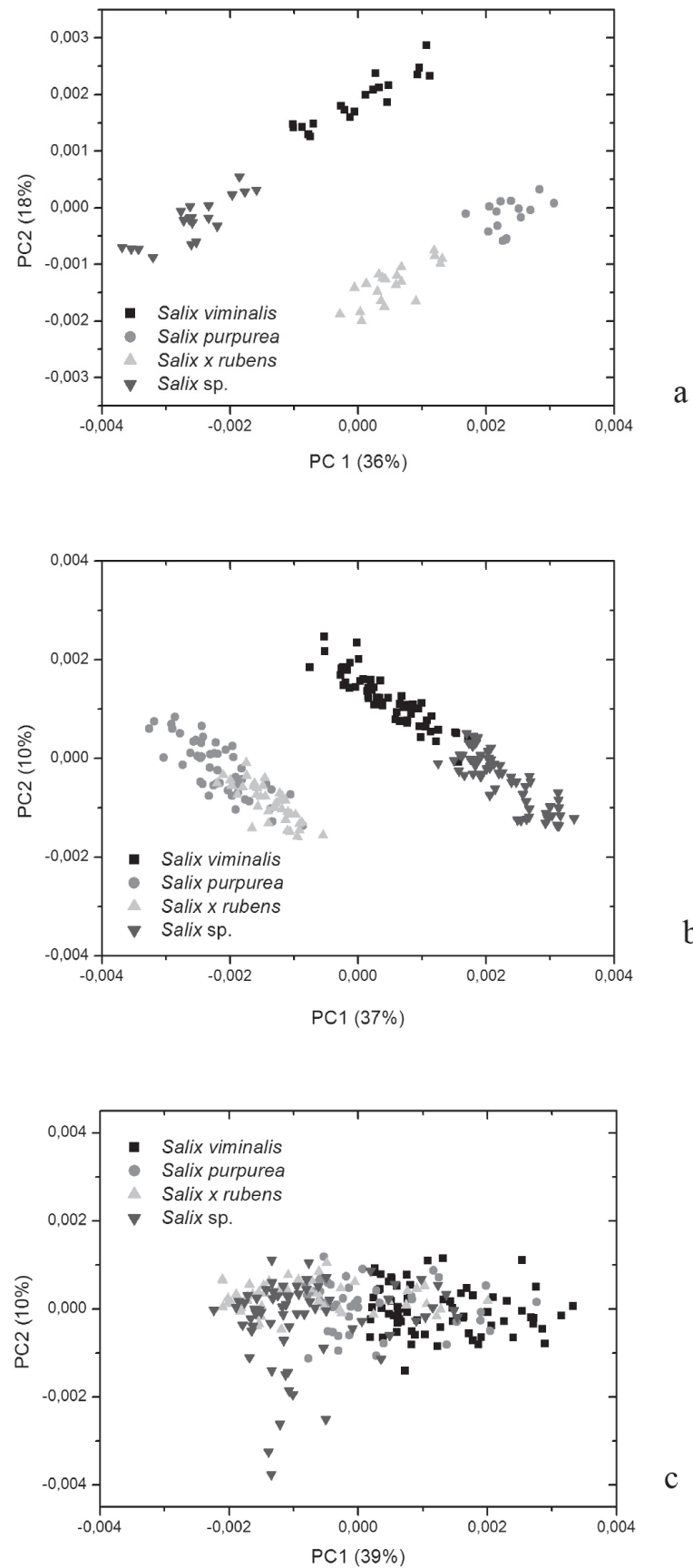

Figura 2. Análisis de componentes principales (PCA), gráfico de scores, (a) Polvo fino, (b) Polvo grueso y (c) Ramas. 
Analizando los gráficos de scores presentados en la figura 2, fue posible observar que prácticamente para todos los análisis el primer y el segundo componente principal, explican cerca del $50 \%$ de la variación, siendo suficiente con estos dos PC's, discriminar las especies, para las muestras de polvo fino y polvo grueso.

Para las muestras de polvo fino (Figura 2a) fue posible identificar una evidente discriminación entre las cuatro especies estudiadas, ya en el análisis con el polvo grueso (Figura 2b) se observa una separación en dos grupos, concluyendo que la mayor granulometría interfiere en la calidad de procesamiento de las informaciones espectrales. Para las muestras de ramas (Figura 2c) no hubo separación de las especies.

Se observa una interferencia por el tipo de muestra en el proceso de discriminación de las especies, siendo el polvo fino, o sea, con menor granulometría, el más indicado. Para los espectros que fueron colectados directamente en las ramas, o sea, en la forma in natura, no hubo una separación de las especies. Hecho justificado por el espectro NIR que puede variar con algunos parámetros físicos, como por ejemplo, tamaño de las partículas, humedad, temperatura y compactación de la muestra (Costa Filho 2003). Cuando una muestra es sometida a una radiación incidente sufre variaciones relativas y dependientes de la constitución química y de los parámetros físicos de las muestras (Davies 2000). Aunque la radiación penetre en el producto, sus características espectrales cambian por causa de la dispersión de la radiación y de los procesos de absorción. En materiales sólidos, la luz penetra en una menor profundidad, la absorción es menor, así las informaciones son mas superficiales. En muestras de polvo, las dimensiones de las partículas facilitan la penetración y la dispersión del infrarrojo en el material y son obtenidas informaciones más detalladas (Hein et al. 2010). Las propiedades de dispersión de la luz están relacionadas con la estructura de la muestra analizada (Nicolai 2007). Así, la menor granulometría, polvo fino, fue el tipo de muestra más indicado para la discriminación entre las especies.

Con los loadings, resultado del análisis por componentes principales, fue factible analizar el "peso" que cada componente principal (PC) recibió e identificar los picos que contribuyen para la clasificación de las especies. Con base en estos resultados se demostró las longitudes de onda más representativas, responsables por la discriminación entre las especies de Salix para el análisis del polvo fino (Figura 3).

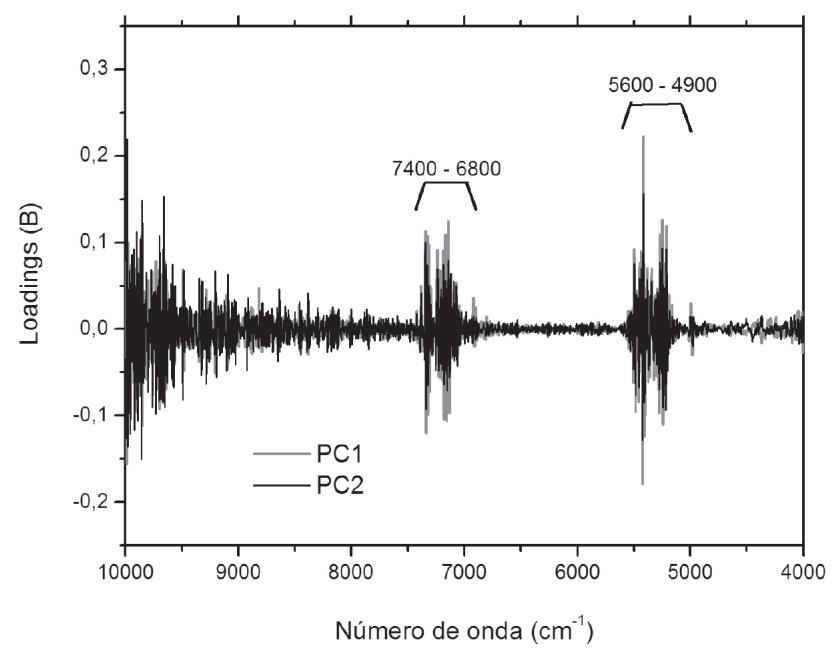

Figura 3. Análisis de componentes principales (PCA), gráfico de loadings, para muestras del polvo fino. 
Para el gráfico de loadings, el PC1, en gris, explica 36\% de la variancia total de los datos en X, el PC2, en negro, explica $18 \%$ de la variancia total de los datos en $\mathrm{X}$, y son los componentes principales responsables por la discriminación entre las especies. Las bandas indicadas entre 7400 y $6800 \mathrm{~cm}^{-1}$ son atribuidas al estiramiento $-\mathrm{OH}$ característicos de la celulosa (Tsuchikawa y Siesler 2003). Los picos indicados entre 5600 y $4900 \mathrm{~cm}^{-1}$ son atribuidos al estiramiento $-\mathrm{CH}$, - $\mathrm{OH}$ combinado, $-\mathrm{CO}$ y estiramiento $-\mathrm{OH}$ con la deformación-OH, atribuidos a la celulosa y agua de impregnación (Siesler et al. 2002, Osborne et al. 1993). La discriminación entre las especies fue factible, principalmente, debido a estas bandas, indicando que el contenido de los componentes químicos, entre otros, proporciona a los vegetales características singulares en cuanto a sus propiedades tecnológicas. Para cada especie de Salix estudiada Nascimento (2009) y Vargas et al. (2012) observaron diferente trabajabilidad y respuestas a los esfuerzos de flexión y tracción con base en su composición anatómica y química.

\section{CONCLUSIONES}

La forma y la granulometría de las muestras de Salix spp. influyeron en la discriminación de las especies por espectroscopía de infrarrojo cercano. Para el material macizo (ramas) la técnica no fue eficiente en virtud de la variación intrínseca de las muestras. La separación de las distintas muestras fue más eficiente para la menor granulometría de las muestras. 


\section{BIBLIOGRAFÍA}

Adedipe, O.E.; Dawsin-Andoh, A.B.; Slahor, J.; Osborn, A.L. 2008. Classification of red oak (Quercus rubra) and white oak (Quercus alba) wood using a near infrared spectrometer and soft independent modeling of class analogies. J Near Infrared Spectroscopy 16(1):49-57.

American Society of Testing and Materials. ASTM. 2012. Standard practices for infrared multivariate, quantitative analysis. ASTM E1655. Vol.03.06. West Conshohocken, Pennsylvania, USA.

Aucott, L.S.; Garthwaite, P.H.; Buckland, S.T. 1988. Transformations to reduce the effect of particle size in near infrared spectra. Analyst 113:1849-1854.

Blanco, M.; Peguero, A. 2008. An expeditious method for determining particle size distribution by near infrared spectroscopy: comparison of PLS2 and ANN models. Talanta 77:647-651.

Braga, J.W.B.; Pastore, T.C.M.; Coradin, V.T.R.; Camargos, J.A.A.; Silva, A.R.D. 2011. The use of near infrared spectroscopy to identify solid wood specimens of Swietenia macrophylla (cites appendix II). Iawa Journal 32(2):285-296.

Casale, M.; Schimleck, L.R.; Espeyd, C. 2010. Classification of pernambuco (Caesalpinia echinata Lam.) wood quality by near infrared spectroscopy and linear discriminant analysis. $J$ Near Infrared Spectroscopy 18(6):435-442.

Costa Filho, P. A. 2003. Estudo comparativo de técnicas de inteligência artificial e modelos lineares em determinações quantitativas no infravermelho próximo, 201f. Tese (doutorado), Universidade Estadual de Campinas - UNICAMP. Campinas, São Paulo.

Davies, A.M.C. 2000. William Herschel and the discovery of near infrared. Spectrosc Eur 12(2): 10-16.

EPAGRI. Sistema para a produção de vime. Florianópolis, 2006. 40p. (Epagri. Sistemas de produção, n.44).

Faix, O.; Böttcher, J.H. 1992. The influence of particle size and concentration in transmission and diffuse reflectance spectroscopy of wood. Holz als Roh-und Werkstoff 50: 221-226.

Hein, P.R.G.; Lima, J.T.; Chaix, G. 2010. Effects of sample preparation on NIR spectroscopic estimation of chemical properties of Eucalyptus urophylla S.T. Blake wood. Holzforschung 64:45-54.

Jones, P.D.; Schimleck, L.R.; Peter, G.F.; Daniels, R.F.; Clark III, A. 2006. Nondestructive estimation of wood chemical composition of sections of radial wood strips by diffuse reflectance near infrared spectroscopy. Wood Science and Technology 40:709-720.

Nascimento, M.B. 2009. Aspectos técnicos e sociais para a sustentabilidade da produção e artesanato do vime. Universidade Federal do Paraná. Tese (Doutorado em Ciências Florestais), UFPR, Curitiba, 247f.

Nicolai, B.M. 2007. Nondestructive measurement of fruit and vegetable quality by means of NIR spectroscopy: A review. Postharvest Biology and Technology 46(2): 99-118.

Osborne, B.G.; Fearn, T.; Hindle, P.H. 1993. Practical NIR Spectroscopy with Applications in Food and Beverage Analysis. 2nd ed., Longman Group, Burnt Mill, Harlow, Essex, England, UK, p. 20-132. 
Otsuka, M. 2004. Comparative particle size determination of phenacetin bulk powder by using KubelkaMunk theory and principal component regression analysis based on near-infrared spectroscopy. Powder Technology 141:244-250.

Pasikatan, M.C.; Steele, J.L.; Spillman, C.K.; Haque, E. 2001. Near infrared reflectance spectroscopy for online particle size analysis of powders and ground materials. J Near Infrared Spectroscopy 9:153-164.

Pastore, T.C.M.; Braga, J.W.B.; Coradin, V.T.R.; Magalhães, W.L.E.; Okino, E.Y.A.; Camargos, J.A.A.; De Muñiz, G.I.B.; Bressan, O.A.; Davrieux, F. 2011. Near infrared spectroscopy (NIRS) as a potential tool for monitoring trade of similar woods: discrimination of true mahogany, cedar, andiroba and curupixá. Holzforschung 65(1):73-80.

Poke, F.S.; Raymond, C.A. 2006. Predicting extractives, lignin, and cellulose contents using near infrared spectroscopy on solid wood in Eucalyptus globulus. Journal of Wood Chemistry and Technology 26(2):187-199.

Punshon, T.; Bertsch, P.M.; Lanzirotti, A.; McLeod, K.; Burger, J. 2003. Geochemical signature of contaminated sediment remobilization revealed by spatially resolved X-ray microanalysis of annual rings of Salix nigra. Environmental Science and Technology 37: 1766-1774.

Russ, A.; Firesova, M.; Gigac, J. 2009. Preliminary study of wood species identification by NIR spectroscopy. Wood Research 54(4): 23-32.

Savitzky, A.; Golay, M. J. E. 1964. Smoothing and differentiation of data by simplified least-squares procedures. Analytical Chemistry 36: 1627-1639.

Sandak, A.; Sandak, J.; Negri, M. 2011. Relationship between near-infrared (NIR) spectra and the geographical provenance of timber. Wood Science and Technology 45(1):35-48.

Schimleck, L.R. 2008. Near infrared spectroscopy: a rapid, non-destructive method for measuring wood properties and its application to tree breeding. New Zealand Journal of Forestry Science 38:14-35.

Schimleck, L.R.; Espey, C.; Mora, C.R.; Evans, R.; Taylor, A.; Muñiz, G.I.B. 2009. Characterization of the wood quality of pernambuco (Caesalpinia echinata Lam.) by measurements of density, extractives content, microfibril angle, stiffness, color and NIR spectroscopy. Holzforschung 63:457-463.

Siesler, H.W; Ozaki, Y.; Kawata, S.; Heise, M. 2002. Near Infrared Spectroscopy: Principle, Instrumentation and Applications. John Wiley-VCH, Weinheim, Germany.

Souza, V.C.; Lorenzi, H. 2005. Botânica sistemática: guia ilustrado para identificação das famílias de Angiospermas da flora brasileira, baseado em APG II. Nova Odessa, SP: Instituto Plantarum.

Tsuchikawa, S.; Schwanninger, M. 2013. A review of recent near-infrared research for wood and paper (Part 2). Applied Spectroscopy Reviews 48:560-587.

Tsuchikawa, S.; Siesler, H.W. 2003. Near-Infrared spectroscopy monitoring of the diffusion process of deuterium-labeled molecules in wood. Part I. Softwood. Appl Spectrosc 57: 667.

Urso, T.; Paieiro, P. 2008. Il legno di salicace: utilizzacioni tradizionali e prospettive future. Atti del Terzo Congresso Nazionale di Selvicoltura. Academia Italiana d Scienze Forestali, Firenze, p.1555-1559. 
Vargas, C.A.; Nascimento, M.B.; Muñiz, G.I.B.; Nisgoski, S. 2012. Physical and mechanical properties of four Salix species. Ciência da Madeira 3(2):80-90.

Vervaeke, P.; Luyssaert, S.; Mertens, J.; Meers, E.; Tack, F.M.G.; Lust, N. 2003. Phytoremediation prospects of willow stands on contaminated sediment: a field trial. Environmental Pollution 126: 275-282.

Zasada, L.; Douglas, D.A.; Buechler, W. 2002. Salix L. Willow. Forest Service's North Central Research, Minnesota. 